\title{
MODULAR LOCALIZATION AND WIGNER PARTICLES
}

\author{
R. BRUNETTI ${ }^{(1)}$ D. GUIDO ${ }^{(2)}$ R. LONGO(2) $^{(2)}$
}

\author{
(1) DIPARTIMENTO DI SCIENZE FISICHE \\ UNIVERSITÀ DI NAPOLI "FEDERICO II" \\ COMPLESSO UNIV. MONTE S. ANGELO \\ I-80126 NAPOLI, ITALY \\ (2) DIPARTIMENTO DI MATEMATICA \\ UNIVERSITÀ DI ROMA "TOR VERGATA" \\ VIA DELLA RICERCA SCIENTIFICA 1 \\ I-00133 ROMA, ITALY
}

Dedicated to Huzihiro Araki on the occasion of his seventieth birthday

\begin{abstract}
We propose a framework for the free field construction of algebras of local observables which uses as an input the Bisognano-Wichmann relations and a representation of the Poincaré group on the one-particle Hilbert space. The abstract real Hilbert subspace version of the Tomita-Takesaki theory enables us to bypass some limitations of the Wigner formalism by introducing an intrinsic spacetime localization. Our approach works also for continuous spin representations to which we associate a net of von Neumann algebras on spacelike cones with the Reeh-Schlieder property. The positivity of the energy in the representation turns out to be equivalent to the isotony of the net, in the spirit of Borchers theorem. Our procedure extends to other spacetimes homogeneous under a group of geometric transformations as in the case of conformal symmetries and de Sitter spacetime.
\end{abstract}

\section{INTRODUCTION}

Although Quantum Physics represents one of the most innovative and drastic conceptual changes of view in modern science, the construction of Quantum Mechanics and Quantum Field Theory has been fruitfully realized with the guidelines of the "classical analogue". This is unsatisfactory, beyond the well known difficulties to construct a quantum field theory with interaction, if one takes the attitude that quantum field theory should stand on its own legs 28].

One point where the structure is selfconsistently dictated by quantum principles is the construction of local observable algebras associated with free fields. We may summarize the construction in the following building blocks:

1. The one-particle Hilbert space.

2. Second quantization.

3. Localization.

Point 1 is E. Wigner's cornerstone analysis of the irreducible unitary representations of (the cover of) the Poincaré group. As is well known, the positive energy representations are classified by the mass $m$ and the spin $s$ if $m>0$. When $m=0$ the stabilizer of a non-zero point is isomorphic to the Euclidean group $E(2)$ which is not compact. Irreducible representations of the Poincaré group induced by finite-dimensional representations of $E(2)$, namely by representations which are

e-mails: brunetti@na.infn.it, guido@mat.uniroma2.it, longo@mat.uniroma2.it.

Supported in part by MIUR and GNAMPA-INDAM. 
trivial on the translational part, are labelled by the helicity (a character on the one-dimensional torus). Irreducible representations of the Poincaré group induced by infinite-dimensional representations of $E(2)$ are historically called continuous spin representations (although properly speaking one should talk of helicity rather than spin). Usually one discards such representations because the corresponding particles have not been experimentally observed so far, but there is no conceptual a priori reason not to consider them. As we will explain below, the analysis in this paper naturally gets into the consideration of the case of continuous spin too.

Point 2 is well described by E. Nelson's expression: "First quantization is a mystery, but second quantization is a functor". Segal's quantization is indeed an automatic procedure to get Weyl operators on the Fock space associated with vectors in the one-particle Hilbert space. In particular one gets a von Neumann algebra out of a real Hilbert subspace of the one-particle space: this is Araki's lattice of von Neumann algebras [1, 2. In this sense free field analysis is basically reduced to one-particle analysis.

In point 3 the basic principle of locality enters. The definition of local real Hilbert subspaces, hence of local von Neumann algebras, requires however one more step. One possibility is to take the functions localized in a region of the configuration spacetime and then get the real Hilbert space in the momentum space. That this procedure is not entirely intrinsic may be seen from the fact that it is not possible to extend it to the case of continuous spin 45 .

The purpose of this note is to show how a net of local algebras may be canonically associated with any positive energy (anti)-unitary representation of the proper Poincaré group. This construction relies on the idea of modular covariance, namely the identification of some one-parameter subgroups of the Poincare group with some modular groups constructed via the Tomita-Takesaki theory. In this way a net of standard subspaces of the representation space may be canonically defined directly in the Wigner one-particle space. Then the second quantization functor produces the net of local von Neumann algebras. Such a net coincides with the one generated by the free Bose field of mass $m$ and spin $s$ when the corresponding irreducible representation of the proper Poincaré group is considered. This construction reveals the deep connection between the positivity of the energy and the isotony property of the net, and reflects the relation between the cyclicity of the vacuum for the intersection of two wedges and the existence of a PCT operator in terms of the Tomita modular conjugations, cf. [22]. Our analysis is related to [5, 35].

In other words, the Bisognano-Wichmann theorem tells us what the Tomita operator associated with a wedge region $W$ should be. Since it is a second quantization operator [15], it is determined by the operator $S_{W}$ on the one-particle Hilbert space $\mathcal{H}$. According to Bisognano-Wichmann

$$
S_{W}=J_{W} \Delta_{W}^{1 / 2}
$$

is made up by the boosts unitaries $\Delta_{W}^{i t}$ and the PCT anti-unitary that are canonically associated with the given (anti)-unitary irreducible representation of the proper Poincaré group. We may then reverse the point of view and define $S_{W}$ by formula (1.1) in terms of the Poincaré group representation, hence define the real subspace

$$
\mathcal{K}_{W} \equiv\left\{\xi: S_{W} \xi=\xi\right\} .
$$

This procedure is, of course, general and can be performed for any unitary representation of the Poincare group, including those with continuous spin, where the construction of the corresponding Wightman fields is not possible 45 .

The von Neumann algebra $\mathcal{R}(W)$ is then defined by

$$
\mathcal{R}(W)=\left\{V(\xi): \xi \in \mathcal{K}_{W}\right\}^{\prime \prime},
$$


where $V$ is the representation of the Weyl commutation relations on the Fock space over $\mathcal{H}$. If $\mathcal{O}$ is a region of the spacetime obtained as intersection of wedges, we may then define

$$
\mathcal{R}(\mathcal{O}) \equiv \bigcap_{W \supset \mathcal{O}} \mathcal{R}(W)
$$

(intersection over all wedges containing $\mathcal{O}$ ). By a classical result the vacuum vector $\Omega$ is cyclic for $\mathcal{R}(\mathcal{O})$ if $\mathcal{O}$ is a double cone, for any irreducible representation of finite helicity. By an intrinsic analysis in terms of Poincaré group representations, we shall show that, in case of continuous spin, $\Omega$ is cyclic for $\mathcal{R}(\mathcal{O})$ if $\mathcal{O}$ is a spacelike cone. But Reeh-Schlieder property for double cones is not to be expected in this case [30].

Our analysis extends to spacetimes with a group of symmetries, where a suitable notion of "wedge region" can be defined, in particular to any such wedge one would associate a one-parameter group of symmetries and a time-reversing reflection, both giving rise to modular objects in the unitary representations. The precise context is explained in Section 5, cf. also [12, 25 for related notions of wedge. Relevant situations are those given by the Minkowski spacetime (or the covering of its Dirac-Weyl compactification) with conformal symmetries, by the circle with Möbius transformations, and by the ( $d$-dimensional) de Sitter spacetime with the isometry group $S O(d, 1)$.

Preliminary versions of this article have been circulating since a few years. The concept of modular localization has then found different applications in papers by B. Schroer and collaborators, see 16 and references therein.

\section{Basic Preliminaries}

Let us recall some basic geometrical and analytical facts. The most important geometrical setting we consider is Minkowski spacetime, but we shall abstract our procedure to extend it to more general spaces and to discuss some other examples.

The Minkowski spacetime is the real manifold $\mathbb{R}^{d} \equiv \mathbb{R} \times \mathbb{R}^{d-1}$ of dimension $d \geq 2$, equipped with the metric

$$
\langle x, y\rangle=x^{0} y^{0}-\sum_{i=1}^{d-1} x^{i} y^{i}, \quad \forall x, y \in \mathbb{R}^{d} .
$$

This makes Minkowski space a Lorentzian manifold and we consider the time orientation fixed once and for all. As a result the Minkowski spacetime is divided into subregions called spacelike, timelike and lightlike corresponding resp. to $\langle x, x\rangle<0$, $\langle x, x\rangle>0$, and $\langle x, x\rangle=0$.

By theorems of Zeeman, the group of diffeomorphisms of the Minkowski space preserving the causal structure is the semidirect product of $\mathbb{R} \times \mathcal{L}$ with the translations, where $\mathbb{R}$ acts as the group of dilations and $\mathcal{L}$ is the full homogeneous Lorentz group. On the other hand the group of isometries of the Minkowski space is the Poincaré group $\mathcal{P}$, the semidirect product $\mathcal{L} \ltimes \mathbb{R}^{d}$, where $\mathbb{R}^{d}$ corresponds to the spacetime translations:

$$
(\Lambda, a) \circ\left(\Lambda^{\prime}, b\right)=\left(\Lambda \cdot \Lambda^{\prime}, a+\Lambda \cdot b\right), \quad \text { with } \Lambda, \Lambda^{\prime} \in \mathcal{L}, a, b \in \mathbb{R}^{d} .
$$

The full Poincaré group $\mathcal{P}$ is simply connected, non connected, non compact, and perfect. It admits a splitting into connected components

$$
\mathcal{P}=\mathcal{P}_{+}^{\uparrow} \cup \mathcal{P}_{+}^{\downarrow} \cup \mathcal{P}_{-}^{\uparrow} \cup \mathcal{P}_{-}^{\downarrow} .
$$

where the \pm corresponds to $\operatorname{det}(g)= \pm 1$, namely selects those transformations which preserve or change the orientation, and the up/down arrow corresponds to 
$\langle x, g x\rangle \gtrless 0$, namely selects those transformations which preserve or change the time orientation.

We shall be mainly concerned with the proper part of the Poincaré group, i.e. $\mathcal{P}_{+}=\mathcal{P}_{+}^{\uparrow} \cup \mathcal{P}_{+}^{\downarrow}$.

Let then $\mathcal{P}_{+} \ni g \longrightarrow U(g)$ be a strongly continuous (anti-)unitary representation on the Hilbert space $\mathcal{H}$, i.e.,

$$
U(g) \text { is } \begin{cases}\text { unitary } & \text { if } g \in \mathcal{P}_{+}^{\uparrow} \\ \text { antiunitary } & \text { if } g \in \mathcal{P}_{+}^{\downarrow}\end{cases}
$$

We select now a particular class of causally complete subregions in Minkowski spacetime which are left globally invariant by suitable one-parameter velocity transformations. It is traditional to call them wedge regions and we denote the set of wedges by $\mathcal{W}$. As usual, $W^{\prime}$ denotes the causal complement of $W$. Each wedge is a Poincaré transform of the wedge $W_{1}=\left\{x \in \mathbb{R}^{d}: x_{1}>\left|x_{0}\right|\right\}$. It is possible to assign to each wedge a one parameter group of transformations $\Lambda_{W}$ and a time-reversing reflection $R_{W}$ satisfying

(a) Reflection covariance. For any $W \in \mathcal{W}, R_{W}$ maps $\mathcal{W}$ onto $\mathcal{W}, R_{W}(W)=$ $W^{\prime}$ and $R_{g W}=g R_{W} g^{-1}, g \in \mathcal{P}_{+}$.

(b) $\Lambda$-covariance. For any $W \in \mathcal{W}, \Lambda_{W}(t)$ maps $\mathcal{W}$ onto $\mathcal{W}, \Lambda_{W}(t)(W)=W$ and $\Lambda_{g W}(t)=g \Lambda_{W}(t) g^{-1}, t \in \mathbb{R}, g \in \mathcal{P}_{+}^{\uparrow}, \Lambda_{g W}(t)=g \Lambda_{W}(-t) g^{-1}, t \in \mathbb{R}$, $g \in \mathcal{P}_{+}^{\downarrow}$.

Indeed, since the action of $\mathcal{P}_{+}$is transitive on the family $\mathcal{W}$, it is enough to choose $\Lambda_{W_{1}}$ and $R_{W_{1}}$ to determine the whole assignment. Moreover, setting $\mathcal{P}_{+}^{\uparrow}(W):=$ $\left\{g \in \mathcal{P}_{+}^{\uparrow}: g W=W\right\}$, properties (a) and (b) imply that $\Lambda_{W}$ is in the center of $\mathcal{P}_{+}^{\uparrow}(W)$, while $R_{W}$ commutes with $\mathcal{P}_{+}^{\uparrow}(W)$.

$\Lambda_{W_{1}}$ is chosen as the (rescaled) boosts preserving $W_{1}$, namely

$$
\Lambda_{W_{1}}: \mathbb{R} \ni t \rightarrow \Lambda_{W_{1}}(t)=\left(\begin{array}{ccccc}
\cosh (2 \pi t) & -\sinh (2 \pi t) & 0 & \ldots & 0 \\
-\sinh (2 \pi t) & \cosh (2 \pi t) & 0 & \ldots & 0 \\
0 & 0 & 1 & \ldots & 0 \\
\vdots & \vdots & \vdots & \ddots & \vdots \\
0 & 0 & 0 & \ldots & 1
\end{array}\right) \in \mathcal{L}_{+}^{\uparrow} .
$$

The element $R_{W_{1}}$ in $\mathcal{P}_{+}$is the reflection w.r.t. the edge of the wedge $W_{1}$, and is given by

$$
R_{W_{1}}\left(x_{0}, x_{1}, \ldots, x_{d-1}\right)=\left(-x_{0},-x_{1}, x_{2}, \ldots, x_{d-1}\right) .
$$

Let's fix a unitary representation $U$ of $\mathcal{P}_{+}$on a Hilbert space $\mathcal{H}$. With $W \in \mathcal{W}$ a wedge, let $H_{W}$ be the self-adjoint generator of $U\left(\Lambda_{W}(t)\right)$ and define

$$
\begin{aligned}
\Delta_{W} & :=\exp \left(H_{W}\right) \\
J_{W} & :=U\left(R_{W}\right) .
\end{aligned}
$$

Proposition 2.1. The following facts hold true:

(i) $\Delta_{W}$ is a densely defined, closed, positive non-singular linear operator on $\mathcal{H}$;

(ii) $J_{W}$ is a antiunitary operator on $\mathcal{H}$ and $J_{W}^{2}=\mathbf{1}$;

(iii) $J_{W} \Delta_{W} J_{W}^{-1}=\Delta_{W}^{-1}$.

Proof. (i) and (ii) are obvious. Concerning (iii), let us observe that $R_{W}$ commutes with $\Lambda_{W}(t)$ which implies that $J_{W} \Delta_{W}^{i t} J_{W}^{-1}=\Delta_{W}^{i t}$, but from the anti-unitarity of $J_{W}$ we have that $J_{W} H_{W} J_{W}^{-1}=-H_{W}$, hence the thesis. 
These properties allow us to introduce and discuss the properties of the following operator

$$
S_{W}:=J_{W} \Delta_{W}^{1 / 2}: \mathcal{H} \longrightarrow \mathcal{H},
$$

indeed, denoting by $R$ and $D$ the range and the domain, we have:

Proposition 2.2. $S_{W}$ is a densely defined, antilinear, closed operator on $\mathcal{H}$ with $R\left(S_{W}\right)=D\left(S_{W}\right)$ and $S_{W}^{2} \subset 1$.

Proof. Density and closedness follow from the corresponding property of $\Delta_{W}$ in Proposition 2.1 $(i)$, antilinearity from the antilinearity of $J_{W}$. Now, $R\left(S_{W}\right) \subset$ $D\left(S_{W}\right) \equiv D\left(\Delta_{W}^{1 / 2}\right)$, indeed by Proposition 2.1 $(i i i)$ we have that $J_{W} \Delta_{W}^{1 / 2} x=$ $\Delta_{W}^{-1 / 2} J_{W} x \in D\left(\Delta_{W}^{1 / 2}\right)$. But we get immediately that $S_{W}^{2}=J_{W} \Delta_{W}^{1 / 2} J_{W} \Delta_{W}^{1 / 2}=$ $\Delta_{W}^{-1 / 2} \Delta_{W}^{1 / 2} \subset 1$ and therefore if $x \in D\left(S_{W}\right)$ then $x=S_{W}\left(S_{W} x\right) \in R\left(S_{W}\right)$, so we can conclude.

Let us now define real subspaces of $\mathcal{H}$ associated with any $W \in \mathcal{W}, \mathcal{K}_{W}=\{h \in$ $\left.D\left(S_{W}\right): S_{W} h=h\right\}$. Recall that an $\mathbb{R}$-linear subspace $G$ in $\mathcal{H}$ is said to be standard whenever the following holds:

$$
\begin{aligned}
G \cap i G & =\{0\}, \\
\overline{G+i G} & =\mathcal{H} .
\end{aligned}
$$

Proposition 2.3. Each $\mathcal{K}_{W}$ is an $\mathbb{R}$-linear closed and standard subspace in $\mathcal{H}, S_{W}$ is the Tomita operator of $\mathcal{K}_{W}$, namely $D\left(S_{W}\right)=\mathcal{K}_{W}+i \mathcal{K}_{W}$ and $S_{W}(h+i k)=h-i k$, $h, k \in \mathcal{K}_{W}$. In particular we have:

$$
\begin{aligned}
\Delta_{W}^{i t} \mathcal{K}_{W} & =\mathcal{K}_{W} \\
J_{W} \mathcal{K}_{W} & =\mathcal{K}_{W}^{\prime},
\end{aligned}
$$

where $\mathcal{K}_{W}^{\prime}:=\left\{h \in \mathcal{H}: \operatorname{Im}(h, k)=0 \quad \forall k \in \mathcal{K}_{W}\right\}$ is the symplectic complement of $\mathcal{K}_{W}$.

Proof. The $\mathbb{R}$-linearity and subspace property of any $\mathcal{K}_{W}$ is obvious. Note first than any $x \in D\left(S_{W}\right)$ can be written as $x=h+i k$ where $h$ resp. $k$ have the form

$$
h=\frac{x+S_{W} x}{2}, \quad k=i \frac{-i\left(x-S_{W} x\right)}{2} .
$$

By the preceding Proposition both terms belong to $\mathcal{K}_{W}$. Hence $\mathcal{K}_{W}+i \mathcal{K}_{W}=$ $D\left(S_{W}\right)$ which is dense, so 2.2) is fulfilled, and if $x \in \mathcal{K}_{W} \cap i \mathcal{K}_{W}$ then $x=S_{W} x$ and $i x=S_{W} i x=-i S_{W} x=-i x$, therefore $x \equiv 0$, and (2.1) holds too.

The graph norm on $D\left(S_{W}\right)$ is, for $x=h+i k$ where $h, k \in \mathcal{K}_{W}$,

$$
\begin{aligned}
\|h+i k\|_{S_{W}}^{2} & =\|h+i k\|^{2}+\left\|S_{W}(h+i k)\right\|^{2} \\
& =\|h+i k\|^{2}+\|h-i k\|^{2} \\
& =2\left(\|h\|^{2}+\|k\|^{2}\right) .
\end{aligned}
$$

Therefore $D\left(S_{W}\right)$ with the graph norm is $\mathcal{K}_{W} \oplus i \mathcal{K}_{W}$, hence the closedness of $\mathcal{K}_{W}$ follows from that of $S_{W}$.

Proposition 2.4. The representation $U$ acts covariantly on the family $\left\{\mathcal{K}_{W}: W \in\right.$ $\mathcal{W}\}$, namely,

$$
U(g) \mathcal{K}_{W}=\mathcal{K}_{g W} \quad g \in \mathcal{P}_{+}^{\uparrow} .
$$


Proof. From properties (a) and (b) it follows that

$$
U(g) \Delta_{W}^{i t} U(g)^{*}=\Delta_{g W}^{i t}
$$

and

$$
U(g) J_{W} U(g)^{*}=J_{g W}
$$

which imply that

$$
U(g) S_{W} U(g)^{*}=S_{g W}
$$

hence the thesis.

Note that eq. (2.3) holds true also for $g \in \mathcal{P}_{+}^{\downarrow}$ due to Prop. 2.3 and the following theorem.

Theorem 2.5. Let $U$ be a (anti-) unitary representation of $\mathcal{P}_{+}$and $W \mapsto \mathcal{K}_{W}$ the above defined map. Then wedge duality holds, namely

$$
\mathcal{K}_{W^{\prime}}=\mathcal{K}_{W}^{\prime} .
$$

Moreover, the following are equivalent:

(i) The spaces $\mathcal{K}_{W}$ are factors, namely $\mathcal{K}_{W} \cap \mathcal{K}_{W}^{\prime}=\{0\}$.

(ii) The representation $U$ does not contain the trivial representation.

(iii) The net is irreducible, namely

$$
\bigcap_{W \in \mathcal{W}} \mathcal{K}_{W}=\{0\}
$$

Proof. Observe that $S_{\mathcal{K}^{\prime}}=S_{\mathcal{K}}^{*}=J_{\mathcal{K}} \Delta_{\mathcal{K}}^{-1 / 2}$. Since $R_{W^{\prime}}=R_{W}$ and $\Lambda_{W^{\prime}}(t)=$ $\Lambda_{W}(-t)$, we get the first statement. Let's prove the equivalences.

$($ ii $) \Rightarrow(i)$. We have $\mathcal{K}_{W} \cap \mathcal{K}_{W^{\prime}}=\mathcal{K}_{W} \cap \mathcal{K}_{W}^{\prime}=\left\{x: U\left(\Lambda_{W}(t)\right) x=x=J_{W} x, \forall t \in \mathbb{R}\right\}$. If such a space contains a non-zero $x$ then the matrix coefficient $(x, U(g) x)$ does not vanish at infinity. By the vanishing of the matrix coefficient theorem for semisimple Lie groups (cf. e.g. 46]) the representation must admit an invariant vector.

$(i) \Rightarrow(i i i)$. This follows directly by the first statement.

$($ iii $) \Rightarrow($ ii $)$. Decompose $\left.U\right|_{\mathcal{P}_{+}^{\uparrow}}$ as $U^{0} \oplus I$ where $I$ is the trivial representation (with some multiplicity) and $U^{0}$ does not contain the trivial representation. The commutation relations between $\Delta^{i t}$ and $J$ imply that any $J$ decomposes accordingly, namely has no anti-diagonal terms. Hence any space $\mathcal{K}_{W}$ decomposes as $\mathcal{K}_{W}=$ $\mathcal{K}_{W}^{0} \oplus \mathcal{K}_{W}^{I}$. We have $U^{I}\left(\Lambda_{W}(t)\right)=I$, and, given two wedges $W_{1}, W_{2}, J_{W_{1}}^{I} J_{W_{2}}^{I}=$ $U^{I}\left(R_{W_{1}} R_{W_{2}}\right)=I$, namely $\mathcal{K}_{W}^{I}$ is independent of $W$. Therefore

$$
\bigcap_{W \in \mathcal{W}} \mathcal{K}_{W} \supset 0 \oplus \mathcal{K}^{I}
$$

Irreducibility implies $\mathcal{K}^{I}=0$, namely $U=U^{0}$.

Remark 2.6. Let us note that the construction of the net $\mathcal{K}_{W}$ requires a representation of $\mathcal{P}_{+}$, or, equivalently, a representation of $\mathcal{P}_{+}^{\uparrow}$ and a PCT operator.

More precisely we need an anti-unitary involution $J$ satisfying $J U(g) J=U(R g R)$, for some space-time reflection $R$. Such involution does not necessarily exist in any representation. However, given a representation $U$ of $\mathcal{P}_{+}^{\uparrow}$ on $\mathcal{H}$, a reflection $R$ and an anti-unitary involution $C$ on $\mathcal{H}$, we may set

$$
\tilde{U}(g)=\left(\begin{array}{cc}
U(g) & 0 \\
0 & C U(R g R) C
\end{array}\right), \quad g \in \mathcal{P}_{+}^{\uparrow}, \quad \tilde{U}(R)=\left(\begin{array}{cc}
0 & C \\
C & 0
\end{array}\right) .
$$

Clearly $\tilde{U}$ gives rise to a (anti)-unitary representation of $\mathcal{P}_{+}$on $\mathcal{H} \oplus \mathcal{H}$. 
Moreover, if $\left.U\right|_{\mathcal{P}_{+}^{\uparrow}}$ is irreducible, then the anti-unitary involution $U\left(R_{W}\right)$ is unique up to a phase, that does not depends on $W$ by covariance. Hence the family $\left\{\mathcal{K}_{W}\right\}$ depends only on $\left.U\right|_{\mathcal{P}^{\uparrow}}$ up to unitary equivalence.

It is known (see e.g. 41]) that a PCT operator exists for an irreducible representation of $\mathcal{P}_{+}^{\uparrow}$ (on $\mathbb{R}^{4}$ ) if and only if the representation is induced by a self-conjugate representation of the stabilizer of a point, which is always the case, except for the finite non-zero helicity representations.

\section{INCLUSIONS OF REAL SUBSPACES AND WEDGES}

Proposition 3.1. Let $\mathcal{K}_{1}, \mathcal{K}_{2}$ be standard subspaces of the Hilbert space $\mathcal{H}$, and assume that $U \mathcal{K}_{1}=\mathcal{K}_{2}$, with $U$ unitary on $\mathcal{H}$. Then $\mathcal{K}_{2} \subset \mathcal{K}_{1}$ iff $\Delta_{1}^{1 / 2} U^{*} \subset$ $J_{1} U^{*} J_{1} \Delta_{1}^{1 / 2}$.

Proof. The following equivalences hold:

$$
\begin{aligned}
\mathcal{K}_{2} \subset \mathcal{K}_{1} & \Longleftrightarrow S_{2} \subset S_{1} \\
& \Longleftrightarrow U J_{1} \Delta_{1}^{1 / 2} U^{*} \subset J_{1} \Delta_{1}^{1 / 2} \\
& \Longleftrightarrow \Delta_{1}^{1 / 2} U^{*} \subset J_{1} U^{*} J_{1} \Delta_{1}^{1 / 2} .
\end{aligned}
$$

The following theorem is a one-particle analogue of results in [8, 43]. It is related to the positive energy criterion in 沟.

Theorem 3.2. Let $\mathcal{K}$ be a standard space in the Hilbert space $\mathcal{H}$ and $U(a)=e^{i a H}$ a one-parameter group of unitaries on $\mathcal{H}$ satisfying

$$
\begin{aligned}
\Delta^{i t} U(a) \Delta^{-i t} & =U\left(e^{\mp 2 \pi t} a\right) \\
J U(a) J & =U(-a),
\end{aligned}
$$

where $J$ and $\Delta$ are the modular conjugation and operator associated with $\mathcal{K}$.

The following are equivalent:

(i) $U(a) \mathcal{K} \subset \mathcal{K}$ for $a \geq 0$;

(ii) $\pm H$ is positive.

Proof. By replacing $\mathcal{K}$ with $\mathcal{K}^{\prime}$ it suffices to prove the case $H$ positive. The implication $(i) \Longrightarrow(i i)$ was proved in 43 .

$(i i) \Longrightarrow(i)$. Let us observe that the spectrum of $H$ is acted upon by the group $\Delta^{i t}$ and by $J$, with $\{0\}$ and $(0, \infty)$ being the invariant subsets. The corresponding eigenspaces are henceforth invariant under the action of $\Delta^{i t}$ and $J$, as a consequence $\mathcal{K}$ is decomposed in a direct sum of respectively the $H=0$ and the $H>0$ parts. Hence the thesis may be proven in the two cases separately. When $H=0$ isotony trivially holds.

In the following we assume that $H>0$. By Proposition 3.1, together with equation (3.2), we get

$$
U(a) \mathcal{K} \subset \mathcal{K} \Longleftrightarrow \Delta^{1 / 2} U(a)^{*} \subset U(a) \Delta^{1 / 2} .
$$

Let $K=\log H$ (it exists since $H>0$ ), and $M$ the generator of $\Delta^{i t / 2 \pi}$. It is easy to see that $e^{i \mu K}$ and $e^{i \lambda M}$ satisfy Weyl's commutation relations, i.e.,

$$
e^{i \lambda M} e^{i \mu K}=e^{i \lambda \mu} e^{i \mu K} e^{i \lambda M} .
$$

According to von Neumann's theorem every representation of the Weyl's commutation relations is equivalent to a multiple of the Heisenberg representation. Then the relation on the right hand side of (3.3) can be checked in just one representation. 
Because of the equivalence (3.3), it is enough to verify the inclusion $U(a) \mathcal{K} \subset \mathcal{K}$, $a>0$, in one non-trivial representation.

An example is provided by the one-particle space of the conformal field theory on the line corresponding to lowest weight representations of $P S L(2, \mathbb{R})$. Taking $\mathcal{K}$ as the standard space associated with the right half-line $(0, \infty)$, and $U(t)$ as the translations, the relations in the hypothesis are verified [10], and the mentioned inclusion of subspaces hold by isotony.

Remark 3.3. Condition (3.2) is not needed for the implication $(i) \Longrightarrow(i i)$ in the above theorem, see [13]. However the condition is necessary for the converse implication. Indeed, given $J, \Delta$ and $U$ as in the theorem, and assuming positivity of the generator of $U(a)$, one may choose a unitary $V$ which commutes with $\Delta$, anticommutes with $J$ and does not commute with $U(a)$, e.g. $V=(\Delta+i)(\Delta-i)^{-1}$, and then replace $J$ with $V J$, the space $\mathcal{K}$ being redefined accordingly. Now property (i) in the theorem above cannot hold, since, by the result of Borchers [8], it would imply condition (3.2) for the new $J$, against the hypothesis.

Let us denote by $H$ the cone in the Lie algebra of $\mathcal{P}_{+}^{\uparrow}$ consisting of the generators of future-pointing light-like or time-like translations. As is known, a unitary representation of $\mathcal{P}_{+}^{\uparrow}$ has positive energy if the corresponding self-adjoint generators are positive. Given two wedges $W_{0} \subset W$, we shall say that $W_{0}$ is positively included in $W$ whenever $W_{0}$ can be obtained by $W$ via a suitable translation $\exp \left(a_{0} h\right), a_{0} \geq 0$, such that $\pm h \in H$, where we denoted by exp the exponential map from the Lie algebra to the Lie group, and

$$
\begin{aligned}
\Lambda_{W}(t) \exp (a h) \Lambda_{W}(-t) & =\exp \left(e^{\mp 2 \pi t} a h\right) \\
R_{W} \exp (a h) R_{W} & =\exp (-a h)
\end{aligned} \quad a, t \in \mathbb{R} .
$$

The following is a well known geometric fact:

(c) Positive inclusion. Any inclusion of wedges is the composition of finitely many positive inclusions.

Theorem 3.4. Let $U$ be a (anti-) unitary representation of $\mathcal{P}_{+}, W_{1} \subset W_{2}$ wedges. Then $\mathcal{K}_{W_{1}} \subset \mathcal{K}_{W_{2}}$ iff $U$ is a positive energy representation.

Proof. Follows immediately from (c) and Theorem 3.2.

Since causally complete convex regions are intersections of wedges, the map $W \rightarrow \mathcal{K}_{W}$ extends to causally complete, convex regions $\mathcal{C}$ via

$$
\mathcal{K}_{\mathcal{C}}=\bigcap_{W \supset \mathcal{C}} \mathcal{K}_{W}
$$

and to general causally complete regions via

$$
\mathcal{K}_{\mathcal{O}}=\bigvee_{\mathcal{C} \subset \mathcal{O}} \mathcal{K}_{\mathcal{C}}
$$

where $\mathcal{C}$ are convex and causally complete. Let us observe that isotony for wedges implies that equation (3.4) is consistent with the original definition of $\mathcal{K}_{W}$.

Denote by $\mathfrak{K}$ the family of all convex causally complete regions. Let us point out he following fact (see e.g. 40]):

(d) Wedge separation. For any space-like separated $\mathcal{O}_{1}, \mathcal{O}_{2} \in \mathfrak{K}$ there exists a wedge $W$ such that $\mathcal{O}_{1} \subset W$ and $\mathcal{O}_{2} \subset W^{\prime}$.

Corollary 3.5. Let $U$ be a positive energy representation of $\mathcal{P}_{+}$. Then the map $\mathcal{O} \longrightarrow \mathcal{K}_{\mathcal{O}}$ is a local Poincaré covariant net of real vector spaces, i.e., isotony holds and if $\mathcal{O}_{1} \subset \mathcal{O}_{2}^{\prime}$ then $\mathcal{K}_{\mathcal{O}_{1}} \subset \mathcal{K}_{\mathcal{O}_{2}}^{\prime}$. If $\mathcal{O}$ is a convex causally complete region then Haag duality holds, namely $\mathcal{K}_{\mathcal{O}^{\prime}}=\mathcal{K}_{\mathcal{O}}^{\prime}$. 
Proof. The first part of the statement holds by definition.

Let us fix $\mathcal{O}_{0} \in \mathfrak{K}$. If $\mathcal{O}_{0}$ is a wedge, then duality has been proved in Theorem 2.5. If $\mathcal{O}_{0}$ is not a wedge, then its space-like complement is not convex, hence, by (d), we have the following chain of identities:

$$
\mathcal{K}_{\mathcal{O}_{0}^{\prime}}^{\prime}=\left(\bigvee_{\substack{\mathcal{O} \subset \mathcal{O}_{0}^{\prime} \\ \mathcal{O} \in \mathfrak{K}}} \mathcal{K}_{\mathcal{O}}\right)^{\prime}=\left(\bigvee_{\substack{W^{\prime} \subset \mathcal{O}_{0}^{\prime} \\ W \in \mathcal{W}}} \mathcal{K}_{W^{\prime}}\right)^{\prime}=\bigcap_{\substack{W^{\prime} \subset \mathcal{O}_{0}^{\prime} \\ W \in \mathcal{W}}} \mathcal{K}_{W^{\prime}}^{\prime}=\bigcap_{\substack{W \supset \mathcal{O}_{0} \\ W \in \mathcal{W}}} \mathcal{K}_{W}=\mathcal{K}_{\mathcal{O}_{0}} .
$$

Therefore Haag duality holds.

Remark 3.6. (1) A net of von Neumann algebras may be obtained via second quantization:

$$
\mathcal{R}(\mathcal{O})=\left\{V(h): h \in \mathcal{K}_{\mathcal{O}}\right\}^{\prime \prime}
$$

where $V(h)$ are the Weyl unitaries on the Bosonic Fock space $e^{\mathcal{H}}$. Weyl unitaries may be defined via

$$
\begin{aligned}
V(h) e^{0} & =e^{-\frac{1}{4}\|h\|^{2}} e^{\frac{i}{\sqrt{2}} h}, \quad h \in \mathcal{H} \\
V(h) V(k) & =e^{-\frac{i}{2} \operatorname{Im}(h, k)} V(h+k) \quad h, k \in \mathcal{H}
\end{aligned}
$$

where the coherent vectors $e^{h}$ are defined by $e^{h}=\bigoplus_{n=0}^{\infty} \frac{h^{\otimes n}}{\sqrt{n !}}$. Coherent vectors turn out to form a total set in $e^{\mathcal{H}}$ (see e.g. [19] p.32), hence the $V(h)$ 's are well defined unitaries. The standard property of $\mathcal{K}_{\mathcal{O}}$ is equivalent to the Reeh-Schlieder property for $\mathcal{R}(\mathcal{O})$ (cf. [1, 15, 33).

(2) If $U$ is the irreducible representation of mass $m$ and spin $s$ the map $\mathcal{O} \longrightarrow$ $\mathcal{R}(\mathcal{O})$ gives the net of local observable algebras for the free field of mass $m$ and spin $s$. In fact, for these nets the one-particle version of the Bisognano-Wichmann theorem holds, i.e.,

$$
\begin{aligned}
J_{W} & =U\left(R_{W}\right) \\
\Delta_{W}^{i t} & =U\left(\Lambda_{W}(t)\right)
\end{aligned}
$$

where $J_{W}$ and $\Delta_{W}$ are the Tomita operators of the real space $\mathcal{K}_{W}$ of vectors localized in $W$. This means that $\mathcal{K}_{W}$ is effectively reconstructed in terms of the representation $U$. Moreover, it was shown by Araki [2] that the map $\mathcal{O} \longrightarrow \mathcal{K}_{\mathcal{O}}$ is an isomorphism of complemented lattices

$$
(\cap, \cup, \text { space-like complement }) \rightleftharpoons(\cap, \vee \text {, symplectic complement })
$$

if $\mathcal{O}$ is connected, causally complete, with piecewise $C^{1}$ boundary. This shows that $\mathcal{K}_{\mathcal{O}}$ is also reconstructed in terms of the representation $U$.

Three questions arise for the subspaces of the described net $\mathcal{O} \rightarrow \mathcal{K}_{\mathcal{O}}$ : the standard property, the $\mathrm{III}_{1}$ factor property (see [3]), namely the fact that the corresponding second quantization algebra is a type $\mathrm{III}_{1}$ factor, and the intersection property (for convex causally complete regions), namely

$$
\mathcal{C}=\bigcap_{i \in I} W_{i} \Rightarrow \mathcal{K}_{\mathcal{C}}=\bigcap_{i \in I} \mathcal{K}_{W_{i}}
$$

When wedge regions are concerned, we proved the standard property, the intersection property and the factor property for irreducible nets. The $\mathrm{III}_{1}$ factor property (for irreducible nets) and the other properties are proved for space like cones in Section 4 . 


\section{INTERSECTIONS AND CYCLICITY}

Proposition 4.1. Let $\mathcal{K}_{j}, j \in \mathcal{J}$, a family of standard subspaces of a Hilbert space $\mathcal{H}$, o a distinguished element of $\mathcal{J}$. Then $\bigcap_{j \in \mathcal{J}} \mathcal{K}_{j}$ is standard if and only if the space

$$
\left\{x \in \mathcal{H}: x \in D\left(S_{j} S_{o}\right) \& S_{j} S_{o} x=x, \forall j \in \mathcal{J}\right\}
$$

is dense.

Proof. Since $\mathcal{K}_{o}$ is standard, $\cap_{j \in \mathcal{J}} \mathcal{K}_{j}$ is standard if and only if $\cap_{j \in \mathcal{J}} \mathcal{K}_{j}+i \cap_{j \in \mathcal{J}} \mathcal{K}_{j}$ is dense. We contend that the last subspace can be equivalently written as the expression in (4.1).

Indeed, if $x \in \mathcal{K}_{j}$ for any $j \in \mathcal{J}$, then $S_{j} x=S_{o} x=x$ for any $j \in \mathcal{J}$. Since range and domain of the $S$ operators coincide, $S_{o} x$ belongs to the domain of $S_{j}$ and $S_{j} S_{o} x=x, j \in \mathcal{J}$. Hence $x$ belongs to the space in (4.1). Such a space being complex linear, it contains also $i \cap_{j \in \mathcal{J}} \mathcal{K}_{j}$.

Conversely, if $x \in D\left(S_{j} S_{o}\right)$ and $S_{j} S_{o} x=x \forall j \in \mathcal{J}$, then, $\forall j \in \mathcal{J}, x \in D\left(S_{j}\right)$, hence it can be written as $x=h_{j}+i k_{j}$ with $h_{j}, k_{j} \in \mathcal{K}_{j}$, and $S_{o} x=S_{j} x$.

Therefore we get $S_{o} x=S_{j} x=S_{j}\left(h_{j}+i k_{j}\right)=h_{j}-i k_{j}$, hence $\frac{1}{2}\left(x+S_{o} x\right)=h_{j}$ and $\frac{1}{2 i}\left(x-S_{o} x\right)=k_{j}$, namely $h_{j}$ and $k_{j}$ are independent of $j$ and belong to $\cap_{j \in \mathcal{J}} \mathcal{K}_{j}$.

Recalling the definition in equation (3.4), we get the following.

Proposition 4.2. Let $U$ be a (anti-) unitary representation of $\mathcal{P}_{+}$on the Hilbert space $\mathcal{H}, \mathcal{C}$ a convex, causally complete region, $W$ a wedge containing $\mathcal{C}$, and $\mathcal{G}(\mathcal{C})=$ $\left\{g \in \mathcal{P}_{+}^{\uparrow}: g W \supset \mathcal{C}\right\}$. Then $\mathcal{K}_{\mathcal{C}}$ is standard iff, denoting by $T(g)$ the operator $\Delta^{-1 / 2} U\left(R g^{-1} R\right) \Delta^{1 / 2}, g \in \mathcal{P}_{+}^{\uparrow}$,

$$
\left\{x \in \mathcal{H}: x \in D(T(g)) \& T(g) x=U\left(g^{-1}\right) x, \forall g \in \mathcal{G}(\mathcal{C})\right\}
$$

is dense, where $\Delta$ and $R$ refer to the wedge $W$.

Also, given two subsets $\mathcal{G}_{1}, \mathcal{G}_{2}$ of $\mathcal{P}_{+}^{\uparrow}, \cap_{\mathcal{G}_{1}} \mathcal{K}_{g W}=\cap_{\mathcal{G}_{2}} \mathcal{K}_{g W}$ iff

$$
\begin{aligned}
\{x \in \mathcal{H}: x \in D(T(g)) \& T(g) x & \left.=U\left(g^{-1}\right) x, \forall g \in \mathcal{G}_{1}\right\} \\
& =\left\{x \in \mathcal{H}: x \in D(T(g)) \& T(g) x=U\left(g^{-1}\right) x, \forall g \in \mathcal{G}_{2}\right\} .
\end{aligned}
$$

Proof. Since the action of $\mathcal{P}_{+}^{\uparrow}$ is transitive on the wedges, the first statement immediately follows by the previous proposition. The second statement follows by the proof of the previous proposition.

Now we may tackle the main questions concerning convex, causally complete regions in the Minkowski space in this approach, namely the standard property (2.1), (2.2), the $\mathrm{III}_{1}$ factor property and intersection property (3.6).

Since local algebras (and local subspaces) are not defined in terms of local fields, the classical Reeh-Schlieder argument does not apply. However, Proposition 4.2 shows that the standardness for a given region (or family of regions) is a property of the representation $U$, hence group theoretic techniques may be applied.

Intersection property instead has to do with the definition in 3.4. Though the local space of a given convex causally complete region $\mathcal{C}$ is defined as the intersection of the spaces of all wedges containing it, just a few of them may be enough to determine $\mathcal{C}$. Would the corresponding intersection of local spaces give rise to the same space? Again, because of the absence of local fields, the answer is not trivial, and the group theoretic approach may do the job. 
Lemma 4.3. Let $U$ be a (anti-)unitary positive energy representation of $\mathcal{P}_{+}$on the Hilbert space $\mathcal{H}, \mathcal{C}$ a convex, causally complete region. Assume that the representation $U$ (restricted to $\mathcal{P}_{+}^{\uparrow}$ ) decomposes as $\int^{\oplus} U_{\lambda} d \mu(\lambda)$. Then $\mathcal{K}_{\mathcal{C}}^{U}$ is standard if and only if $\mathcal{K}_{\mathcal{C}}^{U_{\lambda}}$ is standard for $\mu$-almost all $\lambda$.

Given $W_{j}, j \in \mathcal{J}$, such that $\mathcal{C}=\bigcap_{j \in \mathcal{J}} W_{j}$, then $\mathcal{K}_{\mathcal{C}}^{U}=\bigcap_{j \in \mathcal{J}} \mathcal{K}_{W_{j}}^{U}$ if and only if $\mathcal{K}_{\mathcal{C}}^{U_{\lambda}}=\bigcap_{j \in \mathcal{J}} \mathcal{K}_{W_{j}}^{U_{\lambda}}$ for $\mu$-almost all $\lambda$.

Proof. By Proposition 4.2, both properties depend only on $\left.U\right|_{\mathcal{P}_{+}^{\uparrow}}$. The thesis follows by equations (4.2), (4.3).

Theorem 4.4. Let $U$ be a (anti-) unitary positive energy representation of $\mathcal{P}_{+}$on the Hilbert space $\mathcal{H}, \mathcal{C}$ a spacelike cone. Then the standard property and the intersection property hold. If $U$ does not contain the trivial representation, then the type III $I_{1}$ factor property holds too.

Proof. Let us prove the standard property. Clearly we may assume that the vertex of the space-like cone lies at the origin of the coordinates.

Lemma 4.3 shows that is enough to check the density of the space in 4.2 for all the irreducible positive energy representations. Since this property is known for the positive mass representations and for the zero mass, finite helicity representations, we only have to verify it for the so called continuous spin representations.

Let us now denote by $\mathcal{F}(\mathcal{C})$ the set of wedges containing $\mathcal{C}$. Given a wedge in $\mathcal{F}(\mathcal{C})$, we may consider the family of wedges parallel to the given one and still belonging to $\mathcal{F}(\mathcal{C})$. The intersection of all such wedges is clearly a wedge in $\mathcal{F}(\mathcal{C})$ whose edge contains the vertex of $\mathcal{C}$, namely the origin. Because of isotony (Theorem 3.4),

$$
\bigcap_{W \in \mathcal{F}(\mathcal{C})} \mathcal{K}_{W}=\bigcap_{W \in \mathcal{F}_{0}(\mathcal{C})} \mathcal{K}_{W}
$$

where $\mathcal{F}_{0}(\mathcal{C})$ denotes the subset consisting of wedges whose edge contains the origin.

Then, fixing a wedge $W$ in $\mathcal{F}_{0}(\mathcal{C})$ and setting $\mathcal{G}_{0}(\mathcal{C})=\left\{g \in \mathcal{P}_{+}: g W \in \mathcal{F}_{0}(\mathcal{C})\right\}$, the complex span of the space $\cap_{W \in \mathcal{F}_{0}(\mathcal{C})} \mathcal{K}_{W}$ is given by

$$
\left\{x \in \mathcal{H}: x \in D(T(g)) \& T(g) x=U\left(g^{-1}\right) x, \forall g \in \mathcal{G}_{0}(\mathcal{C})\right\},
$$

namely only the Lorentz subgroup is involved. Therefore the standard property has only to be checked on the restriction to the Lorentz group of the given continuous spin representation $U$. Theorem A.1 concludes the proof.

Let us now prove the intersection property. Again by isotony, we may restrict to the intersection of wedges whose edge contains the origin. If $\mathcal{G}_{1}, \mathcal{G}_{2}$ are two subsets of $\mathcal{L}_{+}^{\uparrow}$ such that $\cap_{g \in \mathcal{G}_{1}} g W=\cap_{g \in \mathcal{G}_{2}} g W=\mathcal{C}$, then the equality $\bigcap_{g \in \mathcal{G}_{1}} \mathcal{K}_{g W}=$ $\bigcap_{g \in \mathcal{G}_{2}} \mathcal{K}_{g W}$ is equivalent to relation (4.3). Then the proof goes on as for the previous case.

We finally prove the $\mathrm{III}_{1}$ factor property. It has been proved in [18 that if 1 is in the spectrum of $\Delta$, but not in the point spectrum, then the second quantization algebra is a type $\mathrm{III}_{1}$ factor. Clearly the property $1 \in \sigma(\Delta) \backslash \sigma_{p}(\Delta)$ is stable under direct sums and quasi-equivalence. Then, by the proof of Theorem A.1, it is enough to show this property for the finite spin representations. Indeed this shows the property for the regular representation of $\mathcal{L}_{+}^{\uparrow}$, hence for the restriction to $\mathcal{L}_{+}^{\uparrow}$ of the continuous spin representations of $\mathcal{P}_{+}^{\uparrow}$, since they are quasi-equivalent to the regular representation.

Now we follow [17], where it is shown (Theorem 3.6) that $\Delta$ can be written as a functional calculus of a selfadjoint operator $B$ via the function $\frac{t+1}{t-1}$, showing in particular that $1 \notin \sigma_{p}(\Delta)$. Moreover, using the explicit formula for $B$, one concludes that $B$ is unbounded, hence 1 is in the spectrum of $\Delta$. 
Now we prove the standard property for light-like strips, namely for regions given by $\bar{W} \cap \overline{W^{\prime}+a}$, where $a$ is a lightlike vector parallel to $W$, namely such that $W+a \subset W$. Such property is motivated by the proof of the spin and statistics property for spacetimes with bifurcated Killing horizon given in [25], Section 4.2.

Theorem 4.5. Let $W$ and $a$ be as above and assume the spacetime dimension is $d \neq 2$. For any positive energy (anti-) unitary representation of $\mathcal{P}_{+}, \mathcal{K}_{\bar{W} \cap \overline{W^{\prime}+a}}$ is standard.

Proof. Clearly any wedge containing $L=\bar{W} \cap \overline{W^{\prime}+a}$ either contains $W$ or contains $W^{\prime}+a$. Then, by isotony (Theorem 3.4),

$$
\bigcap_{W \supset L} \mathcal{K}_{W}=\mathcal{K} \cap \mathcal{K}_{W^{\prime}+a}
$$

Let us assume for the moment that $U$ is the trivial representation. Then translations and boosts act trivially, namely $\mathcal{K}_{W^{\prime}+a}=\mathcal{K}_{W^{\prime}}=\mathcal{K}_{W}$, since $S_{W^{\prime}}=S_{W}^{*}=S_{W}$. Therefore we may assume that $U$ does not contain the trivial representation, namely $U$ does not have invariant vectors. Since $d \neq 2$, the vanishing of the matrix coefficient theorem applies (cf. e.g. [46], Proposition 2.3.5), hence the spectrum of the generator of any light-like translation is strictly positive, i.e. zero is not an eigenvalue.

As explained before, the standard property is equivalent to the density of the space

$$
\left\{x \in D \left(\Delta^{1 / 2} U\left(\tau((a)) \Delta^{1 / 2}\right): U\left(\tau((a)) \Delta^{1 / 2} U\left(\tau((a)) \Delta^{1 / 2} x=x\right\},\right.\right.\right.
$$

where $\tau(a)$ denotes the translation by $a$. This property clearly depends only on the restriction of the representation of the Poincaré group to the subgroup $\mathbb{P}_{1}$ generated by boosts and light-like translations with strictly positive generator (relative to the wedge $W$ ). As the logarithm of the generator of translations and the generator of the boosts give rise to (and are determined by) a representation of the CCR in one dimension, the strictly positive energy representations of $\mathbb{P}_{1}$ have a simple structure: they are always a multiple of the unique irreducible representation. Therefore the density of the space in eqn. (4.5) holds either always or never, and hence can be checked in the irreducible case. But this is the case of the current algebra on the circle, where cyclicity holds by conformal covariance.

Now we show that some form of the intersection property holds for double cones too.

Let $\mathcal{C}$ be a diamond generated by a relatively open convex subregion $\Omega$ of some space-like hyperplane $\mathcal{G}$. For any $\xi \in \partial \Omega$, let us consider the family $\mathcal{F}(\xi)$ of the half-spaces in $\mathcal{G}$ tangent to $\Omega$ at $\xi$, namely the half-spaces containing $\Omega$ and whose boundary contains $\xi$. Being parametrized by the normal vectors at $\xi$, they have a linear structure, and clearly form a closed convex set. Let us denote by $\mathcal{F}_{*}(\xi)$ its extreme points, and by $\mathcal{F}_{*}(\Omega)$ the union $\bigcup_{x \in \partial \Omega} \mathcal{F}_{*}(\xi)$. Clearly $\Omega=\bigcap_{h \in \mathcal{F}_{*}(\Omega)} h$. We shall call $\mathcal{F}_{*}(\Omega)$ the minimal family for $\Omega$. Analogously, denoting with $W_{h}$ the wedge generated by the space-like half-space $h$, we shall call $\mathcal{F}_{*}(\mathcal{C})=\left\{W_{h}: h \in \mathcal{F}_{*}(\Omega)\right\}$ the minimal family for $\mathcal{C}$. Clearly when $\mathcal{C}$ is the intersection of a finite number of wedges $W_{i}$, the minimal family $\mathcal{F}_{*}(\mathcal{C})$ consists only of (some) $W_{i}$.

Theorem 4.6. Let $\mathcal{C}$ be a diamond generated by a relatively open convex subregion $\Omega$ of some space-like hyperplane $\mathcal{G}, \mathcal{F}_{*}(\mathcal{C})$ its minimal family. Then

$$
\mathcal{K}_{\mathcal{C}}=\bigcap_{W \in \mathcal{F}_{*}(\mathcal{C})} \mathcal{K}_{W}
$$


Proof. Let $W$ be a wedge containing $\mathcal{C}$. Then $W \cap \mathcal{G} \supset \Omega$. Since $W \cap \mathcal{G}$ is a cone given by the intersection of (at most) two half spaces $h_{1}, h_{2}$ of $\mathcal{G}$, then, by the intersection property for space-like cones, one gets $\mathcal{K}_{W} \supset \mathcal{K}_{W_{h_{1}} \cap W_{h_{2}}} \supset \mathcal{K}_{\mathcal{C}}$ and $\mathcal{K}_{W_{h_{1}}} \cap \mathcal{K}_{W_{h_{2}}}=\mathcal{K}_{W_{h_{1}} \cap W_{h_{2}}}$. Therefore

$$
\mathcal{K}_{\mathcal{C}}=\bigcap_{h \in \mathcal{F}(\Omega)} \mathcal{K}_{W_{h}}
$$

Then, again by the intersection property for space-like cones, for any point $\xi \in \partial \Omega$, we may replace $\cap_{h \in \mathcal{F}(\xi)} \mathcal{K}_{W_{h}}$ with $\cap_{h \in \mathcal{F}_{*}(\xi)} \mathcal{K}_{W_{h}}$, since $\cap_{h \in \mathcal{F}(\xi)} W_{h}$ is a spacelike cone, and the proof is completed.

Theorem 4.7. The following pair of classes can be put in one-to-one correspondence:

(i) Positive energy representations of $\mathcal{P}_{+}$.

(ii) Local nets of closed real vector spaces on $\mathfrak{K}$ satisfying modular covariance, namely $\Delta_{W}^{i t} \mathcal{K}_{\mathcal{O}}=\mathcal{K}_{\Lambda_{W}(t) \mathcal{O}}$, and standard property for the space-like cones.

Proof. The map from $(i)$ to $(i i)$ has been illustrated above. The inverse map has been constructed in [11], getting a representation of the universal covering of $\mathcal{P}_{+}^{\uparrow}$. It has been shown in [22] that such representation is indeed a representation of $\mathcal{P}_{+}^{\uparrow}$, and extends to a representation of $\mathcal{P}_{+}$.

Remark 4.8. Let $U$ be a unitary representation of $\mathcal{P}_{+}^{\uparrow}$ on a Hilbert space $\mathcal{H}$ which is finite direct sum of irreducible representations each with strictly positive mass. As recently shown in [36], if $\mathcal{F}: W \in \mathcal{W} \rightarrow \mathcal{F}_{W}$ is a net of standard real subspaces of $\mathcal{H}$ and $U$ acts covariantly on $\mathcal{F}$, namely $U(g) \mathcal{F}_{W}=\mathcal{F}_{g W}$, then $\mathcal{F}_{W}$ is the standard subspace associated with $W$ and $U$.

\section{FreE NETS ON DIFFERENT SPACETIMES}

In this section we discuss various extensions of the previous construction to different spacetimes. We begin with a general setting.

Let $M$ be a globally hyperbolic spacetime, $G$ a (Lie) group of transformations acting on it (e.g. isometries, or conformal transformations), $G_{+}$the subgroup of orientation preserving transformations, $G^{\uparrow}$ the subgroup of time-preserving transformations, $G_{+}^{\uparrow}$ their intersection.

Assume it is possible to choose a triple $(\mathcal{W}, R, \Lambda)$ where $\mathcal{W}$ is a family of open, causally complete subregions, called wedges, stable under the action of $G_{+}, R$ : $W \rightarrow R_{W}$ is a map from $\mathcal{W}$ to time-reversing reflections in $G_{+}, \Lambda: W \rightarrow \Lambda_{W}$ is a map from $\mathcal{W}$ to one-parameter subgroups of $G_{+}^{\uparrow}$ satisfying the following properties:

(a) Reflection covariance. For any $W \in \mathcal{W}, R_{W}$ maps $\mathcal{W}$ onto $\mathcal{W}, R_{W}(W)=$ $W^{\prime}$ and $R_{g W}=g R_{W} g^{-1}, g \in G_{+}$.

(b) $\Lambda$-covariance. For any $W \in \mathcal{W}, \Lambda_{W}(t)$ maps $\mathcal{W}$ onto $\mathcal{W}, \Lambda_{W}(t)(W)=W$ and $\Lambda_{g W}(t)=g \Lambda_{W}(t) g^{-1}, t \in \mathbb{R}, g \in G_{+}^{\uparrow}, \Lambda_{g W}(t)=g \Lambda_{W}(-t) g^{-1}, t \in \mathbb{R}$, $g \in G_{+}^{\downarrow}$.

Remark 5.1. Properties (a) and (b) imply that $R_{W^{\prime}}=R_{W}$ and $\Lambda_{W^{\prime}}(t)=\Lambda_{W}(-t)$. Moreover, if $g W=W$, then $g$ commutes with $\Lambda_{W}$ and $R_{W}$, namely $\Lambda_{W}$ belongs to the center of the stabilizer $G_{+}^{\uparrow}(W)=\left\{g \in G_{+}^{\uparrow}: g W=W\right\}$ and $R_{W}$ commutes with $G_{+}^{\uparrow}(W)$.

If $G_{+}^{\uparrow}$ acts transitively on $\mathcal{W}$, then the assignments $W \rightarrow \Lambda_{W}, W \rightarrow R_{W}$ are determined by the choice of a one parameter subgroup in the center of the stabilizer of one wedge $W_{0}$, and by the choice of a reflection commuting with $G_{+}^{\uparrow}\left(W_{0}\right)$. 
In many cases, e.g. Minkowski spacetime with Poincaré symmetry in dimension $d \neq 3$, or Minkowski with conformal symmetry in any dimension, or de Sitter spacetime in dimension $d \neq 3$, the center of $G_{+}^{\uparrow}(W)$ is one-dimensional, hence $\Lambda_{W}$ is fixed up to rescaling.

Given a (anti)-unitary representation $U$ of $G_{+}$, we can reproduce the analysis in Section2: Set $\Delta_{W}=U\left(\Lambda_{W}(-i)\right), J_{W}=U\left(R_{W}\right)$ (the above normalization at $t=-i$ is conventional, as we could arbitrarily rescale $\Lambda_{W}$. The positive energy condition, see below, will fix the normalization). Clearly $J_{W}$ is a self-adjoint antiunitary, and $\Delta$ is strictly positive. By (a) and (b), $R_{W}=R_{\Lambda_{W}(t) W}=\Lambda_{W}(t) R_{W} \Lambda_{W}(-t)$, namely $R_{W}$ and $\Lambda_{W}$ commute. Therefore $J_{W} \Delta_{W} J_{W}=\Delta_{W}^{-1}$, and, setting $S_{W}=J_{W} \Delta_{W}^{1 / 2}$, we easily obtain that $S_{W}$ is closed, densely defined and satisfies $S_{W}^{2} \subset I$.

Set $\mathcal{K}_{W}=\left\{\xi \in D\left(S_{W}\right): S_{W} \xi=\xi\right\}$. It turns out that $\mathcal{K}_{W}$ is a standard space, and that the representation $U$ acts geometrically on the family: $U(g) \mathcal{K}_{W}=\mathcal{K}_{g W}$. Moreover, essential duality holds: $\mathcal{K}_{W}^{\prime}=\mathcal{K}_{W^{\prime}}$.

Let $\mathcal{B}$ be the family of regions that are intersections of wedges, and set $\mathcal{K}_{B}=$ $\cap_{W \supset B} \mathcal{K}_{W}, B \in \mathcal{B}$. If we assume $\mathcal{W}$ to be a subbase for the topology of $M$, then $\mathcal{B}$ forms a base, hence any open set $\mathcal{O}$ is a union of elements in $\mathcal{B}$. Then we may define $\mathcal{K}_{\mathcal{O}}=\vee_{B \subset \mathcal{O}} \mathcal{K}_{B} . G$-covariance follows as in Section 2 .

Proposition 5.2. The following properties hold:

(i) $\left\{\mathcal{K}_{W}, W \in \mathcal{W}\right\}$ is a covariant family of real subspaces, namely $\mathcal{K}_{g \mathcal{W}}=U(g) \mathcal{K}_{\mathcal{W}}$, $g \in G_{+}$, moreover $\mathcal{K}_{W}$ is standard and $\mathcal{K}_{W^{\prime}}=\mathcal{K}_{W}^{\prime}$.

(ii) $\left\{\mathcal{K}_{B}, B \in \mathcal{B}\right\}$ is a covariant net of real subspaces, namely $B_{1} \subset B_{2}$ implies $\mathcal{K}_{B_{1}} \subset \mathcal{K}_{B_{2}}$, and $\mathcal{K}_{g \mathcal{B}}=U(g) \mathcal{K}_{\mathcal{B}}, g \in G_{+}$.

Remark 5.3. As in Remark 2.6, giving a representation of $G_{+}$is equivalent to giving a representation of $G_{+}^{\uparrow}$ together with some sort of PCT, namely an anti-unitary involution $J$ satisfying $J U(g) J=U(R g R)$, for some reflection $R$.

Notice that the net $B \rightarrow \mathcal{K}_{B}, B \in \mathcal{B}$, is not necessarily local. Also, it is not necessarily true that $\mathcal{K}_{B}=\mathcal{K}_{W}$ if $B=W$, namely it may happen that $\cap_{W} \supset W_{0} \mathcal{K}_{W}$ is strictly smaller than $\mathcal{K}_{W}$, since we did not prove wedge isotony. We need further assumptions to solve these two problems.

Let $H$ be a convex cone in the Lie algebra of $G$, and let us denote by exp the exponential map from the Lie algebra to $G_{+}^{\uparrow}$. If $W_{0} \subset W$ are wedges, we shall say that $W_{0}$ is positively included in $W$ w.r.t the cone $H$ if there is a one parameter subgroup $\exp (a h)$ of $G_{+}^{\uparrow}$, depending on $W_{0}$ and $W$, with $\exp \left(a_{0} h\right) W=W_{0}$ for some $a_{0} \geq 0$, such that $\pm h \in H$, and

$$
\begin{aligned}
\Lambda_{W}(t) \exp (a h) \Lambda_{W}(-t) & =\exp \left(e^{\mp 2 \pi t} a h\right) \\
R_{W} \exp (a h) R_{W} & =\exp (-a h)
\end{aligned} \quad a, t \in \mathbb{R} .
$$

Let us assume the following:

(c) Positive inclusion. Any inclusion of wedges is the composition of finitely many positive inclusions.

(d) Wedge separation. For any space-like separated $\mathcal{O}_{1}, \mathcal{O}_{2} \in \mathcal{B}$ there exists a wedge $W$ such that $\mathcal{O}_{1} \subset W$ and $\mathcal{O}_{2} \subset W^{\prime}$.

We shall say that a (anti)-unitary representation of $G_{+}$is positive if, whenever $h \in H$, the self-adjoint generators in the representation space of the one-parameter groups $U(\exp (a h))$ are positive.

Theorem 5.4. Assume the triple $(\mathcal{W}, R, \Lambda)$ satisfies assumptions (a), (b), (c), (d), and let $U$ be a (anti)-unitary positive representation of $G_{+}$. Then wedge isotony holds, namely $W_{1} \subset W_{2}$ implies $\mathcal{K}_{W_{1}} \subset \mathcal{K}_{W_{2}}$, the net $B \rightarrow \mathcal{K}_{B}, B \in \mathcal{B}$, is 
local and extends the net $W \rightarrow \mathcal{K}_{W}, W \in \mathcal{W}$. Moreover, for any $B \in \mathcal{B}$ such that $B^{\prime} \notin \mathcal{B}$, Haag duality holds:

$$
\mathcal{K}_{B}^{\prime}=\mathcal{K}_{B^{\prime}}
$$

If $G_{+}^{\uparrow}$ is a simple Lie group with finite center and $U$ does not contain the trivial representation, the net is irreducible. If moreover the closure of $\left\{\Lambda_{W}(t): t \in \mathbb{R}\right\}$ in $G_{+}^{\uparrow}$ is not compact, the local space $\mathcal{K}_{W}$ is a factor.

Proof. Wedge isotony follows by property (c), locality of $B \mapsto \mathcal{K}_{B}$ follows by property (d). If $B_{0}^{\prime} \notin \mathcal{B}$ then $\mathcal{K}_{B_{0}^{\prime}}=\cup_{B \subset B_{0}^{\prime}} \mathcal{K}_{B}$, hence Haag duality follows as in Corollary 3.5. The assumption of non-compactness for the closure of $\Lambda(t)$ allows us to use the vanishing of the matrix coefficients Theorem as in Theorem 2.5 to prove the factoriality.

Let us observe that, if the positivity in the previous statement is a non-trivial requirement, namely if there are wedges included one in another, then $\Lambda$ and $\exp (a h)$ give rise to a representation of the $a x+b$ group, namely the requirement that the closure of $\left\{\Lambda_{W}(t): t \in \mathbb{R}\right\}$ in $G_{+}^{\uparrow}$ is not compact is automatically satisfied. For the same reason, also the assumption on the finiteness of the center is unnecessary (cf. 23]).

Let us discuss a toy example satisfying the general scheme presented above, where the last statement of the previous Theorem does not apply. Let $M=S^{2} \times$ $\mathbb{R}$, where $S^{2}$ is the unit sphere in $\mathbb{R}^{3}$, with the induced Lorentzian metric, and $G_{+}=S O(3) \times \mathbb{R} \rtimes \mathbb{Z}_{2}$, where $S O(3)$ acts on the sphere, $\mathbb{R}$ gives time-translations, and the $\mathbb{Z}_{2}$ element implements the orientation preserving space-time reflection (PT transformation). We also set $\mathcal{W}$ to be the family of diamonds with base a hemisphere (at time $t$ ). Clearly the stabilizer $G_{+}^{\uparrow}(W)$ is one-dimensional, and, since two hemispheres included one in the other coincide, no positivity is needed. Therefore the parametrization of the groups $\Lambda_{W}$ may be fixed arbitrarily. Also, the action of $G_{+}$is transitive, hence we may fix a wedge $W_{0}$ as the causal completion of $\left\{(t, x, y, z): x^{2}+y^{2}+z^{2}=1, t=0, z>0\right\}$ and assign

$$
\Lambda_{W_{0}}(\theta)=\left(\begin{array}{cccc}
1 & 0 & 0 & 0 \\
0 & \cos \theta & \sin \theta & 0 \\
0 & -\sin \theta & \cos \theta & 0 \\
0 & 0 & 0 & 1
\end{array}\right),
$$

$R_{W_{0}}(t, x, y, z)=(-t, x, y,-z)$. In any faithful irreducible representation of $G_{+}^{\uparrow}$, the generator of $\Lambda_{W_{0}}$ has a one-dimensional kernel, therefore the corresponding space $\mathcal{K}_{W_{0}}$ is not a factor. More precisely it is a tensor product of a continuous abelian von Neumann algebra and of a type $I_{\infty}$ factor (cf. [18]). However the net is irreducibile.

In such a generality, it is not possible to prove important properties, such as the standard property, the intersection property, or the factor property, for elements of $\mathcal{B}$. We now discuss this structure in specific spacetimes.

5.1. Conformal Group. In the following the conformal group on the Minkowski spacetime $M$ of dimension $d \geq 1$ (with $M=\mathbb{R}$ if $d=1$ ) is the group generated by the Poincaré group ( $a x+b$ " group if $d=1$ ) and the relativistic ray inversion map. The conformal group is isomorphic to $P S O(d, 2)$. If $d>2$, this is the group of local diffeomorphisms (defined out of meager sets) which preserve the metric tensor up to non-vanishing functions; its universal covering acts globally and transitively on the universal covering of the Dirac-Weyl compactification of $M$. If $d=2$, the DiracWeyl compactification is a two-torus, and only the time-covering is considered, namely the conformal group acts on the cylinder spacetime with non compact time 
curves. In the $d=1$ case the identity component of $P S O(d, 2)$ is isomorphic to $P S L(2, \mathbb{R})$ and we consider its action on $S^{1}$. For details see 10$]$.

If $d \geq 2$ a wedge is any conformal transformed of (the lift of) a wedge in the Minkowski space, in particular Poincaré-wedges, double cones, future cones and past cones give rise to conformal wedges. The maps $R$ and $\Lambda$ are here the lifts of those defined on Minkowski spacetime. If $d=1$ wedges are proper intervals, the reflection associated with the upper semi-circle maps $z \in S^{1}$ to its complex conjugate $\bar{z}$, and $\Lambda$ is the (lift of the) one parameter subgroup of $\operatorname{PSL}(2, \mathbb{R})$ of (Cayley transformed) dilations.

Then, we may consider (anti-)unitary representations of these groups, and check that properties (a), (b) and (c) hold true, the cone $H$ being generated by the Lie algebra generators of lightlike translations and their conjugates under the action of the conformal group. In this case wedges form already a base for the topology, so it is enough to consider the net on wedges. Hence assumption (d) is not needed. An analogue of Theorem 5.4 holds true here.

Theorem 5.5. Let the spacetime be the universal covering of the compactified Minkowski space $S^{d-1} \times \mathbb{R}$ for $d \geq 2$ and $S^{1}$ if $d=1$. Let $U$ be a (anti-)unitary positive representation of $\widetilde{P S O}(d, 2)$ which does not contain the trivial representation. Then, $\mathcal{W} \ni W \rightarrow \mathcal{K}_{W}$ is a local conformal net for which Haag duality holds. The standard and the III factor properties are satisfied. Moreover, the family is irreducible.

Proof. Haag duality, conformal covariance and standard property follow by Proposition $5.2(i)$, wedge-isotony, factor property and irreducibility follow by Theorem 5.4, and locality follows by wedge isotony and wedge-duality. $\mathrm{III}_{1}$ factor property follows as in Proposition 1.2, 23].

The one-dimensional conformal case is extensively studied in [26] and we refer to that paper for further details. We recall that all the nets corresponding to irreducible representations of $P S L(2, \mathbb{R})$ are subsystems ( $n$-th derivatives) of the same net on $\mathbb{R}$ (the $U(1)$ current algebra) which is their common dual net.

5.2. de Sitter spacetime. Since the $d$-dimensional de Sitter spacetime $d S^{d}$ may be defined as the hyperboloid $x_{0}^{2}+1=\sum_{i=1}^{d} x_{i}^{2}$ in $M^{d+1}$, the wedges can be defined as the intersection of this hyperboloid with the wedges in $M^{d+1}$ whose edges contains the origin.

The natural symmetry group of $d S^{d}$ is the Lorentz group $\mathcal{L}_{+}=S O(d, 1)$, and the maps $R, \Lambda$ are assigned here as in the Minkowski spacetime. Then properties (a), (b), and (d) immediately follow from the corresponding properties for the Minkowski spacetime. Property (c) instead is trivially satisfied, since two wedges $W_{1} \subset W_{2}$, whose edge contain the origin, coincide.

Intersections of wedges, namely elements of $\mathcal{B}$, correspond to spacelike cones in the Minkowski space, therefore the standard property and the intersection property on the $d$-dimensional de Sitter spacetime can be studied applying the techniques of the preceding section. But we can also rely on the direct analysis by Bros and Moschella [9].

Let us recall that the irreducible representations of the group $S O(d, 1), d \geq 2$, belong to three classes, usually called principal series representations, complementary series representations, and discrete series representations (cf. e.g. 42, 38]). The first class corresponds to representations appearing in the direct integral decomposition of the regular representation, the second one to representations not appearing in the direct integral decomposition of the regular representation. Concerning the third class however, the name "discrete series" is not always appropriate, namely 
it is not always true that they are irreducible direct summands of the regular representation. Indeed, applying a result of Harish-Chandra, it turns out that this fact is possible if and only if $d$ is even (see [38|). The exact determination of the direct-summand representations, namely the recognition of the discrete series as opposed to the "mock discrete series" is well known for the two-covering $S L(2, \mathbb{R})$ of $S O(2,1)$ 32], implying that there are not mock discrete series representations for $S O(2,1)$. This problem has been solved in [14] for $d=4$ and in [39] for a general $d=2 m, m \geq 2$.

Theorem 5.6. Let $\{\mathcal{K}(B): B \in \mathcal{B}\}$ be the net of local real vector subspaces associated to a representation $U$ of the Lorentz group $S O(d, 1)$. If $U$ is a subrepresentation of the regular representation, then the standard property, the intersection property and the factor property hold. If $U$ is a representation in the principal or complementary series of the Lorentz group $S O(d, 1)$, then the mentioned properties hold.

Proof. The restriction of a representation from the Poincaré group to the Lorentz group gives a map from nets $\mathcal{K}^{M}$ on the Minkowski space $M^{d}$ to nets $\mathcal{K}^{S}$ on the de Sitter space defined as $\mathcal{K}^{S}(B)=\mathcal{K}^{M}(\mathcal{C}(B))$, where $\mathcal{C}(B)$ is the spacelike cone in $M^{d}$ generated by the region $B$ in $S^{d-1}$ and the origin. We may rephrase results in the previous section saying that the standard property, the intersection property and the factor property hold for regions $B$ given as intersections of wedges in the regular representation of the Lorentz group.

By Theorem A.1, cf. also Remark A.8, the properties hold for all subrepresentations. Since the regular representation decomposes as direct integral of the principal series representations, the standard and the intersection properties hold for almost all values of the parameter labeling the principal series.

However, we may use the analysis in [9], where it is shown that a class of free fields may be constructed, corresponding to the principal, resp. complementary series of the representations of $\mathcal{L}_{+}^{\uparrow}$. In [9] the authors prove the Reeh-Schlieder and Bisognano-Wichmann properties for the free fields corresponding to the principal series, and state that these results extend to the complementary series. By the Bisognano-Wichmann property, such free fields necessarily give rise to the nets constructed as above for the corresponding representations. Therefore the standard property follows by the Reeh-Schlieder property and the intersection property is trivially satisfied since the local algebras are generated by local fields.

Thus, concerning the principal and complementary series, the standard and intersection properties are consequence of the Reeh-Schlieder in [9]. Yet the above proof goes beyond that, by showing the same properties to hold in the $d S^{d}$ models associated with the discrete series, $d$ even. Discrete series representations have been explicitly excluded in the analysis in [9], and the result that such representations give rise to (free) nets of local algebras on the de Sitter space-time seems to be not known before our analysis. This will be discussed in detail in [27].

\section{Appendix A. Restricting the Poincaré group Representations to the LORENTZ SUBGROUP}

We give here an analysis of the representations of the Lorentz and Poincaré groups needed in the paper. We treat explicitly the $3+1$-dimensional case, however the analysis extends to any dimension, as explained in Remark A.8.

If $G$ is a locally compact group, we shall denote by $\lambda_{G}$ its left regular representation. If $H \subset G$ is a closed subgroup and $\pi$ is a unitary representation of $H$, we shall denote by $\operatorname{Ind}_{H \uparrow G}(\pi)$ the representation of $G$ induced by $\pi$ in the sense 
of Frobenius, Wigner and Mackey; we shall refer to the books 446, 31, 34 for the theory of induced representations.

Let us recall that the irreducible representations of $\mathcal{P}_{+}^{\uparrow}$ are induced representations $\operatorname{Ind}_{F \uparrow \mathcal{P}_{+}^{\uparrow}}(\eta)$, where $F=F(p)$ is the stabilizer of some point $p \in \mathbb{R}^{4}, \mathcal{P}_{+}^{\uparrow}$ acting on the subgroup $\mathbb{R}^{4}$ by conjugation, and $\eta$ is an irreducible representation of $F$. We often identify $\mathbb{R}^{4}$ with its dual $\hat{\mathbb{R}}^{4}$. When $p$ varies in a given $\mathcal{P}_{+}^{\uparrow}$-orbit the corresponding induced representations are equivalent, therefore they are labelled by $m=p^{\mu} p_{\mu}$. When $m>0$ the stabilizer is isomorphic to $S O(3) \ltimes \mathbb{R}^{4}$, therefore positive mass $m$ representations are completely described by the spin $s$. When $m=0$ and we choose $p_{0}>0$ (to have positive energy), the stabilizer is isomorphic to the Euclidean group $E(2)$. The representations which are trivial on the $E(2)$-translations are the so-called finite-helicity representations, and are completely labelled by $\mathbb{Z}$. The others are called continuous-spin representations.

The other cases, namely $p=0$ and $m<0$, correspond respectively to null energy (trivial translations) and non positive energy.

In the following we shall say that a property $P$ for representations of a group $G$ is stable if " $P$ is true for $\pi$ " implies " $P$ is true for all representations unitarily equivalent to $\pi$ " and

$$
P \text { is true for } \pi \equiv \pi_{1} \oplus \pi_{2} \Leftrightarrow P \text { is true for } \pi_{1} \text { and } \pi_{2} .
$$

Theorem A.1. Assume that $P$ is a stable property for the representations of $\mathcal{L}_{+}^{\uparrow}$. The following are equivalent:

(i) $P$ is true for the restriction to $\mathcal{L}_{+}^{\uparrow}$ of the positive mass representations.

(ii) $P$ is true for the restriction to $\mathcal{L}_{+}^{\uparrow}$ of the continuous spin representations.

(iii) $P$ is true for the restriction to $\mathcal{L}_{+}^{\uparrow}$ of the massless finite helicity representations.

(iv) $P$ is true for $\lambda_{\mathcal{L}_{+}^{\uparrow}}$.

The proof of this theorem requires some steps.

Lemma A.2. Let $\pi=\operatorname{Ind}_{F \uparrow \mathcal{P}_{+}^{\uparrow}}(\eta)$ be an irreducible representation of $\mathcal{P}_{+}^{\uparrow}$ as above, $F=F\left(p_{0}\right)$. Then

$$
\left.\pi\right|_{\mathcal{L}_{+}^{\uparrow}}=\operatorname{Ind}_{E \uparrow \mathcal{L}_{+}^{\uparrow}}\left(\left.\eta\right|_{E}\right),
$$

where $E$ is the stabilizer, in $\mathcal{L}_{+}^{\uparrow}$, of $p_{0}$

Proof. Let us denote by $X$ the orbit of $p_{0}$ under the adjoint action of $\mathcal{P}_{+}^{\uparrow}$ on the subgroup $\mathbb{R}^{4}$, or equivalently the homogeneous space $\mathcal{P}_{+} / F\left(p_{0}\right)$, and let $\nu$ be the $\mathcal{P}_{+}^{\uparrow}$-invariant measure on $X$. If $\eta$ is a representation of $F$ acting on the Hilbert space $\mathcal{H}_{\eta}$ of $\eta, \pi$ can be defined as

$$
(\pi(g) \xi)(p)=\eta(\alpha(g, p)) \xi\left(g^{-1} p\right)
$$

where $g \in \mathcal{P}_{+}^{\uparrow}, p \in X, \xi \in L^{2}\left(X, \mathcal{H}_{\eta}, d \nu\right)$, and $\alpha$ is an $F$-valued cocycle of the form $\alpha(g, p)=s(p)^{-1} g s\left(g^{-1} p\right)$, where $s$ is a Borel section, namely a Borel map $s: X \rightarrow \mathcal{P}_{+}^{\uparrow}$ satisfying $s(p) p_{0}=p$.

By definition, $\mathbb{R}^{4}$ acts trivially on itself, hence $F=E \ltimes \mathbb{R}^{4}$ with $\mathbb{R}^{4}$ acting trivially on $X$, therefore we may choose $s$ to be a $\mathcal{L}_{+}^{\uparrow}$-valued section. As a consequence, $\alpha: \mathcal{L}_{+}^{\uparrow} \times X$ is $E$-valued, namely the restriction to $\mathcal{L}_{+}^{\uparrow}$ of $\pi$ is by definition the representation induced by $\left.\eta\right|_{E}$.

If $\rho$ and $\sigma$ are representations, we shall write $\rho=\sigma$ if $\rho$ is unitary equivalent to $\sigma$ and $\rho \approx \sigma$ if $\rho$ is quasi equivalent to $\sigma$, namely $\rho \otimes \iota=\sigma \otimes \iota$, where $\iota$ is the identity representation on $\ell^{2}(\mathbb{N})$. 
Lemma A.3. Let $H$ be a locally compact group isomorphic to the Euclidean group $E(2)$. If $\pi$ is an irreducible unitary representation of $H$ and $\pi$ has non-trivial restriction to the subgroup $\mathbb{R}^{2}$, then $\pi=\pi_{q} \equiv \operatorname{Ind}_{\mathbb{R}^{2} \uparrow H}(q)$ where $q \neq 0$ is a character $q \in \hat{\mathbb{R}}^{2}$.

We have $\lambda_{H}=\int_{\mathbb{R}^{2}}^{\oplus} \pi_{q} d q$.

Proof. $E(2)$ is the semidirect product $E(2)=\mathbb{R}^{2} \rtimes \mathbb{T}$, where $\mathbb{T}$ acts on the plane $\mathbb{R}^{2}$ by rotations. The action of $E(2)$ on $\hat{\mathbb{R}}^{2}$ by dual conjugation factors through the action of $\mathbb{T}$ and is smooth. The stabilizer $H_{q}$ of a point $q \in \hat{\mathbb{R}}^{2}$ is $E(2)$ (iff $q=0$ ) or $H_{q}=\mathbb{R}^{2}$. By Mackey's theorem every irreducible representation $\pi$ of $H$ is induced from an irreducible representation $\rho$ of $H_{q}$ with $\left.\rho\right|_{\mathbb{R}^{2}}=\operatorname{dim}(\rho) q$.

Thus either $q=0$ and $\pi$ acts trivially on $\mathbb{R}^{2}$, or $q \neq 0$ and $\pi=\pi_{q}$.

The rest is now clear by induction at stages because

$$
\lambda_{H}=\operatorname{Ind}_{\mathbb{R}^{2} \uparrow H}\left(\lambda_{\mathbb{R}^{2}}\right)=\operatorname{Ind}_{\mathbb{R}^{2} \uparrow H}\left(\int_{\hat{\mathbb{R}}^{2}}^{\oplus} q \mathrm{~d} q\right)=\int_{\hat{\mathbb{R}}^{2}}^{\oplus} \operatorname{Ind}_{\mathbb{R}^{2} \uparrow H}(q) \mathrm{d} q=\int_{\hat{\mathbb{R}}^{2}}^{\oplus} \pi_{q} \mathrm{~d} q .
$$

Proposition A.4. Let $G$ be a locally compact group and $H \subset G$ a closed subgroup isomorphic to the Euclidean group E(2). Then

$$
\lambda_{G}=\int_{\hat{\mathbb{R}}^{2}}^{\oplus} \operatorname{Ind}_{H \uparrow G}\left(\pi_{q}\right) d q .
$$

Proof. Immediate by the Lemma A.3 because

$$
\lambda_{G}=\operatorname{Ind}_{H \uparrow G}\left(\lambda_{H}\right)=\operatorname{Ind}_{H \uparrow G}\left(\int_{\hat{\mathbb{R}}^{2}}^{\oplus} \pi_{q} \mathrm{~d} q\right)=\int_{\hat{\mathbb{R}}^{2}}^{\oplus} \operatorname{Ind}_{H \uparrow G}\left(\pi_{q}\right) \mathrm{d} q .
$$

We shall denote by $\pi_{m, s}$ the irreducible representation of mass $m>0$ and spin $s \in \mathbb{N}$ of the Poincaré group $\mathcal{P}_{+}^{\uparrow}$ and by $\pi_{m, s}^{\mathcal{L}_{+}^{\uparrow}}$ its restriction to the Lorentz subgroup $\mathcal{L}_{+}^{\uparrow}$.

By definition, the continuous spin representations $\sigma_{q}$ of $\mathcal{P}_{+}^{\uparrow}$ are the ones induced by the representations $\pi_{q}$ of $H=E(2) \ltimes \mathbb{R}^{4}$ in Lemma A.3, where $H$ is the stabilizer in $\mathcal{P}_{+}^{\uparrow}$ of a point $p$ with $\langle p, p\rangle=0, p_{0}>0$. We shall denote by $\sigma_{q}^{\mathcal{L}_{+}^{\uparrow}}$ the restriction of $\sigma_{q}$ to $\mathcal{L}_{+}^{\uparrow}$. By Lemma A.2 we have $\sigma_{q}^{\mathcal{L}_{+}^{\uparrow}}=\operatorname{Ind}_{\mathbb{R}^{2} \uparrow \mathcal{L}_{+}^{\uparrow}}(q)$.

Lemma A.5. $\lambda_{\mathcal{L}_{+}^{\uparrow}}=\int_{\mathbb{\mathbb { R }}^{2}}^{\oplus} \sigma_{q}^{\mathcal{L}_{+}^{\uparrow}} d q$.

Proof. Immediate by Lemmas A.2, A.3 and Prop. A.4.

Lemma A.6. For any given $m>0$ we have

$$
\lambda_{\mathcal{L}_{+}^{\uparrow}} \approx \bigoplus_{s \in \mathbb{N}} \pi_{m, s}^{\mathcal{L}_{+}^{\uparrow}}
$$

Proof. Denote by $\rho_{s}$ the representation of $S O(3)$ of spin s. By Lemma A.2 $\pi_{m, s}^{\mathcal{L}_{+}^{\uparrow}} \approx$ $\operatorname{Ind}_{S O(3) \uparrow \mathcal{L}_{+}^{\uparrow}}\left(\rho_{s}\right)$, in particular $\pi_{m, s}^{\mathcal{L}_{+}^{\uparrow}}$ is independent of $m>0$.

We have

$$
\bigoplus_{s \in \mathbb{N}} \pi_{m, s}^{\mathcal{L}_{+}^{\uparrow}}=\bigoplus_{s \in \mathbb{N}} \operatorname{Ind}_{S O(3) \uparrow \mathcal{L}_{+}^{\uparrow}}\left(\rho_{s}\right)=\operatorname{Ind}_{S O(3) \uparrow \mathcal{L}_{+}^{\uparrow}}\left(\bigoplus_{s \in \mathbb{N}} \rho_{s}\right) \approx \operatorname{Ind}_{S O(3) \uparrow \mathcal{L}_{+}^{\uparrow}}\left(\lambda_{S O(3)}\right) \approx \lambda_{\mathcal{L}_{+}^{\uparrow}}
$$


The following Lemma is a particular case of the subgroup Theorem of Mackey (cf. e.g. [34], Chapter II, Theorem 1) when the subgroup $G_{2}$ coincides with the group $G$. We give a proof here for the convenience of the reader.

Lemma A.7. Let $H$ be a subgroup of $G, \eta$ a representation of $H$ and $g_{0}$ an element of $G$ normalizing $H$. Then

$$
\operatorname{Ind}_{H \uparrow G}(\eta)=\operatorname{Ind}_{H \uparrow G}\left(\eta^{g_{0}}\right),
$$

where $\pi^{g_{0}}(h) \equiv \pi\left(g_{0}^{-1} h g_{0}\right), h \in H$.

Proof. Let us denote by $X$ the homogeneous space $G / H$, and let $\nu$ be a $G$-quasiinvariant measure on $X$, that for simplicity we assume to be invariant. Setting $\pi=\operatorname{Ind}_{H \uparrow G}(\eta)$, namely

$$
(\pi(g) \xi)(p)=\eta(\alpha(g, p)) \xi\left(g^{-1} p\right),
$$

where $g \in G, p \in X, \xi \in L^{2}\left(X, \mathcal{H}_{\eta}, d \nu\right)$, and $\alpha$ is an $H$-valued cocycle $\alpha(g, p)=$ $s(p)^{-1} g s\left(g^{-1} p\right)$, with $s: X \rightarrow G$ a Borel section satisfying $s(p) p_{0}=p$.

Hence $\pi^{g_{0}}$ is given by

$$
\begin{aligned}
\left(\pi^{g_{0}}(g) \xi\right)(p)= & \eta\left(g_{0}^{-1} \alpha(g, p) g_{0}\right) \xi\left(g^{-1} p\right) \\
& =\eta\left(g_{0}^{-1} s(p)^{-1} g s\left(g^{-1} p\right) g_{0}\right) \xi\left(g^{-1} p\right)=\eta\left(\alpha^{g_{0}}(g, p)\right) \xi\left(g^{-1} p\right),
\end{aligned}
$$

where the cocycle $\alpha^{g_{0}}(g, p)=g_{0}^{-1} s(p)^{-1} g s\left(g^{-1} p\right) g_{0}=s^{g_{0}}(p)^{-1} g s^{g_{0}}\left(g^{-1} p\right)$ is associated with the map $s^{g_{0}}(p)=s(p) g_{0}$. Clearly $s^{g_{0}}: X \rightarrow G$ is a Borel section for the different quotient map $g \rightarrow g g_{0}^{-1} p_{0}$. As the stabilizer of $p_{0}$ coincides with the stabilizer of $g_{0}^{-1} p_{0}$, the statement follows by the uniqueness of the induced representation.

Proof. (of Theorem A.A) (i) $\Leftrightarrow(i v)$ : By Lemma A.6, property $P$ holds for the representations $\pi_{m, s}^{\mathcal{L}_{+}^{\uparrow}}$ iff it holds for the regular representation, by stability.

$(i i) \Leftrightarrow(i v)$ : If $p \neq 0$ has zero mass, the stabilizer $E(p)$ in $\mathcal{L}_{+}^{\uparrow}$ does not change replacing $p$ with $\lambda p, \lambda>0$. Therefore all elements in $\mathcal{L}_{+}^{\uparrow}$ moving $p$ to some of its multiples normalizes the stabilizer of $p$. For such a $g$,

$$
\pi_{q}\left(g^{-1} h g\right)=\pi_{g q}(h), h \in E(p) .
$$

Note that every $p$-orbit in $\hat{\mathbb{R}}^{2}$ (except $\{0\}$ ) can be reached by some $g$ with the property $g: p \mapsto \lambda p$. Therefore the $\sigma_{q}^{\mathcal{L}_{+}^{\uparrow}}$ 's are all equivalent, by Lemma A.7. Then, by Lemma A.5, $\sigma_{q}^{\mathcal{L}_{+}^{\uparrow}}$ is a subrepresentation of the regular representation, hence property $P$ holds by stability. The converse is also true by stability.

(iii) $\Leftrightarrow\left(\right.$ iv): The argument is again similar to the above ones. Let $\chi_{n} \in \hat{\mathbb{T}}, n \in \mathbb{Z}$ be the characters of $\mathbb{T}$. The finite helicity representations are the representations of the Poincaré group induced by the representations $\alpha_{n} \equiv \operatorname{Ind}_{\mathbb{T} \uparrow E(2)}\left(\chi_{n}\right)$ of $E(2)$. Their restrictions to $\mathcal{L}_{+}^{\uparrow}$ are $\operatorname{Ind}_{E(2) \uparrow \mathcal{L}_{+}^{\uparrow}}\left(\alpha_{n}\right)$. Then, by induction at stages,

$$
\bigoplus_{n} \underset{E(2) \uparrow \mathcal{L}_{+}^{\uparrow}}{\operatorname{Ind}}\left(\alpha_{n}\right)=\bigoplus_{n} \underset{\mathbb{T} \uparrow \mathcal{L}_{+}^{\uparrow}}{\operatorname{Ind}}\left(\chi_{n}\right)=\underset{\mathbb{T} \uparrow \mathcal{L}_{+}^{\uparrow}}{\operatorname{Ind}}\left(\bigoplus_{n} \chi_{n}\right)=\underset{\mathbb{T} \uparrow \mathcal{L}_{+}^{\uparrow}}{\operatorname{Ind}}\left(\lambda_{\mathbb{T}}\right)=\lambda_{\mathcal{L}_{+}^{\uparrow}},
$$

and the statement follows by stability.

Remark A.8. Although the proof of Theorem A.1 has been written for the 4dimensional case, it extends to the case of the Poincaré group $\mathcal{P}_{+}^{\uparrow}(d)$ acting on the $d$-dimensional Minkowski space, $d \geq 2$. Indeed the continuous spin representations are present only when $d \geq 4$, therefore the property $(i i)$ is void for dimension $\leq 3$. When $d \geq 4$, the stabilizer of a light-like point is the Euclidean group $E(d-2)$, 
whose irreducible representations are parametrized by vectors in $\mathbb{R}^{d-2}$ (and vectors with the same length give equivalent representations). This can be found e.g. in [42], or proved by induction where the first is given by Lemma A.3 and the induction step follows by the Mackey Theorem (46], Thm 7.3.1). Therefore all the above analysis applies.

\section{REFERENCES}

[1] H. Araki, A lattice of von Neumann algebras associated with the quantum field theory of a free Bose field, J. Math. Phys. 4 (1963), 1343.

[2] H. Araki, von Neumann algebras of local observables for free scalar field, J. Math. Phys. $\mathbf{5}$ (1964), 1-13.

[3] H. Araki, Type of von Neumann algebra associated with free field, Progr. Theoret. Phys. 32 (1964), 956-965.

[4] P. Bertozzini, R. Conti, R. Longo, Covariant sectors and positivity of the energy, Commun. Math. Phys. 141 (1998), 471-492.

[5] H. Baumgärtel, M. Jurke, F. Lledó, On free nets over the Minkowski spacetime, Rep. Math. Phys. 35 (1995), 101-127.

[6] J. Bisognano, E. Wichmann, On the duality condition for a Hermitean scalar field, J. Math. Phys. 16 (1975), 985.

[7] J. Bisognano, E. Wichmann, On the duality condition for quantum fields, J. Math. Phys. 17 (1976), 303-321.

[8] H.J. Borchers, The CPT theorem in two-dimensional theories of local observables, Commun. Math. Phys. 143 (1992), 315.

[9] J. Bros, U. Moschella, Two-point functions and quantum fields in de Sitter universe, Rev. Math. Phys. 8 (1996), 327-391.

[10] R. Brunetti, D. Guido, R. Longo, Modular structure and duality in conformal quantum field theory, Commun. Math. Phys. 156 (1993) 201-219.

[11] R. Brunetti, D. Guido, R. Longo, Group cohomology, modular theory and space-time symmetries, Rev. Math. Phys. 7 (1994), 57-71.

[12] D. Buchholz, O. Dreyer, M. Florig, S.J. Summers, Geometric modular action and spacetime symmetry groups, Rev. Math. Phys. 12 (2000) 475-560.

[13] D. Buchholz, S.J. Summers, An algebraic characterization of vauum states in Minkowski space, Commun. Math. Phys. 155 (1993) 449-458.

[14] J. Dixmier, Représentations intégrables du groupe de De Sitter. Bull. Soc. Math. France 89 (1961) 9-41.

[15] J.P. Eckmann, K. Osterwalder, An application of Tomita's theory of modular Hilbert algebras: Duality for free Bose fields, J. Funct. Anal. 13 (1973), 1-22.

[16] L. Fassarella, B. Schroer, Wigner particle theory and local quantum physics, hep-th/0112168.

[17] F. Figliolini, D. Guido, The Tomita operator for the free scalar field, Ann. Inst. H. Poincaré Phys. Théor. 51 (1989), 419-435.

[18] F. Figliolini, D. Guido, On the type of second quantization factors, J. Operator Th. 31 (1994), 229-252.

[19] A. Guichardet, Tensor products of $C^{*}$-algebras (infinite), Aarhus Univ., Lecture notes series, 13 (1969).

[20] D. Guido, R. Longo, Relativistic invariance and charge conjugation in quantum field theory, Commun. Math. Phys. 148, (1992) 521.

[21] D. Guido, Modular covariance, PCT, spin and statistics, Ann. Ist. H. Poincaré, 63, 383 (1995).

[22] D. Guido, R. Longo, An algebraic spin and statistics theorem, Commun. Math. Phys. 172 (1995), 517-533.

[23] D. Guido, R. Longo, The conformal spin and statistics theorem, Commun. Math. Phys. 181, (1996) 11.

[24] D. Guido, R. Longo, Natural energy bounds in quantum thermodynamics, Commun. Math. Phys. 218 (2001), 513-536.

[25] D. Guido, R. Longo, J.E. Roberts, R. Verch, Charged sectors, spin and statistics in quantum field theory on curved space-times, Rev. Math. Phys. 13 (2001), 125-198

[26] D. Guido, R. Longo, H.-W. Wiesbrock, Extensions of conformal nets and superselection structures, Commun. Math. Phys. 192, 217 (1998)

[27] D. Guido, R. Longo, Work in progress.

[28] R. Haag "Local Quantum Physics", Springer-Verlag, New York-Berlin-Heidelberg 1996.

[29] P. Hislop, R. Longo, Modular structure of the local observables associated with the free massless scalar field theory, Commun. Math. Phys. 84 (1982), 71-85. 
[30] G.J. Iverson, G. Mack, Quantum fields and interaction of massless particles: the continuous spin case, Ann. of Phys. 64 (1971) 211-253.

[31] A.A. Kirillov "Elements of the Theory of Representations", Springer-Verlag, BerlinHeidelberg 1976.

[32] S. Lang " $S L_{2}(R)$ ", Springer-Verlag, New York-Berlin-Heidelberg 1985.

[33] P. Leyland, J.E. Roberts, D. Testard, Duality for the free electromagnetic field, Marseille preprint 1976, unpublished.

[34] R.L. Lipsman "Group Representations", LNM 338, Springer-Verlag, Berlin-Heidelberg-New York 1974.

[35] F. Lledó, Conformal covariance of massless free nets, Rev. Math. Phys. 13 (2001), 11351161.

[36] J. Mund, The Bisognano-Wichmann theorem for massive theories, Ann. Henri Poincaré 2 (2001), 907-926.

[37] S. Stratila, L. Zsido "Lectures on von Neumann Algebras" Abacus Press, 1979.

[38] E.A. Thieleker, The unitary representations of the generalized Lorentz groups, Trans. Amer. Math. Soc. 199 (1974), 327-367.

[39] E.A. Thieleker, On the integrable and square-integrable representations of $\operatorname{Spin}(1,2 m)$, Trans. Amer. Math. Soc. 230 (1977), 1-40.

[40] L.J. Thomas, E.H. Wichmann. On the causal structure of Minkowski spacetime J. Math. Phys. 38 (1997) 5044-5086.

[41] V. S. Varadarajan, "Geometry of Quantum Theory", Springer-Verlag, New York, 1985.

[42] N.Ja. Vilenkin, A.U. Klimyk "Representation of Lie groups and special functions. Vol. 2. Class I representations, special functions, and integral transforms" Mathematics and its Applications (Soviet Series), 74. Kluwer Academic Publishers Group, Dordrecht, 1993

[43] H.-W. Wiesbrock, A comment on a recent work of Borchers, Lett. Math. Phys. 25 (1992) 157-159.

[44] H.-W. Wiesbrock, Half-sided modular inclusions of von Neumann algebras, Commun. Math. Phys. 157 (1993), 83.

[45] D. Yngvason, Zero-mass infinite spin representations of the Poincaré group and quantum field theory, Commun. Math. Phys. 18 (1970), 195-203.

[46] R.J. Zimmer "Ergodic Theory of Semisimple Lie Groups" Birkhäuser, Boston-BaselStuttgart 1984. 